\title{
Increased derived neutrophil-to-lymphocyte ratio and Breast Imaging-Reporting and Data System classification predict poor survival in patients with non-distant metastatic HER2+ breast cancer treated with neoadjuvant chemotherapy
}

This article was published in the following Dove Press journal: Cancer Management and Research

Yuyong $\mathrm{Li}^{1, *}$

Yi Shao ${ }^{2, *}$

Lishan $\mathrm{Bai}^{3}$

Xingwei Zhou ${ }^{4}$

'Department of Radiology, The Third People's Hospital of Bengbu, Bengbu, Anhui, China; ${ }^{2}$ Department of Ophthalmology, The First Affiliated Hospital of Nanchang University, Nanchang, Jiangxi, China; ${ }^{3}$ Department of General Surgery, The Third People's Hospital of Bengbu, Bengbu, Anhui, China; ${ }^{4}$ Department of Ultrasound, Ankang Hospital of Traditional Chinese Medicine, Ankang, Shanxi, China

*These authors contributed equally to this work
Correspondence: Xingwei Zhou Department of Ultrasound, Ankang Hospital of Traditional Chinese Medicine, No 47 Bashan East Road, Ankang, Shanxi, 725000 , China

Email Zxw6I2421@I63.com
Introduction: The prognostic role of the derived neutrophil-to-lymphocyte ratio (dNLR) in human patients with HER2+ breast cancer is not well understood. Here, we aimed to investigate the prognostic significance of dNLR in patients with HER2+ breast cancer undergoing neoadjuvant chemotherapy.

Methods: A total of 310 patients with non-distant metastatic HER2+ breast cancer who had received neoadjuvant chemotherapy in our hospital from May 2006 to November 2013 were retrospectively included in this study. Kaplan-Meier curves were used to assess overall survival (OS) and disease-free survival (DFS). The Cox regression model was used to evaluate the prognostic value of dNLR and Breast Imaging-Reporting and Data System (BI-RADS) classification, as well as other clinicopathological parameters in patients with HER2+ breast cancer treated with neoadjuvant chemotherapy.

Results: We found that dNLR prior to treatment was positively correlated with tumor size, tumor stage, lymphovascular invasion, and histological grade $(P<0.05)$. The median OS of patients with high dNLR and low dNLR were 44.2 and 69.9, respectively $(P<0.001)$, and the median DFS of patients with high dNLR and low dNLR were 15.3 and 22.1 months, respectively $(P<0.001)$. Multivariate analysis showed that dNLR was an independent risk factor for OS $(\mathrm{HR}=1.726 ; 95 \% \mathrm{CI}: 1.072-2.662 ; P=0.009)$ and DFS (HR $=1.658 ; 95 \%$ CI: $1.125-2.426$; $P=0.026$ ). Moreover, increased BI-RADS classification independently predicted short OS (HR $=1.609 ; 95 \%$ CI: $1.216-2.351 ; P=0.015)$ and DFS (HR $=1.925 ; 95 \%$ CI: $1.526-2.635 ; P=0.021)$.

Conclusion : dNLR prior to treatment and BI-RADS classification are independent prognostic factors in patients with HER2+ breast cancer receiving neoadjuvant chemotherapy.

Keywords: breast cancer, inflammation, dNLR, Breast Imaging-Reporting and Data System, prognosis

\section{Introduction}

Breast cancer is the most common malignancy among women worldwide, including China. ${ }^{1}$ Overexpression of HER2 is a poor prognostic factor in patients with breast cancer. Currently, drugs that target HER2, such as herceptin, lapatinib, ado-trastuzumab, emtansine, and pertuzumab, are primarily used to treat HER2+ breast cancer, thereby increasing the survival and quality of life of patients. However, the number of deaths per year due to HER2+ breast cancer remains high. ${ }^{1}$ HER2+ breast cancer frequently 
recurs after first-line treatment, resulting in metastasis and ultimately death. ${ }^{2}$ Therefore, it is particularly important to predict the prognosis of patients with HER2+ breast cancer. At present, prognostic factors in these patients mainly include age, tumor size, histological grade, and lymph node status. Although several novel prognostic factors, such as circulating-free DNA, lymphocyte infiltration, and circulating tumor cells, have been discovered in recent years, ${ }^{3-6}$ the clinical application of these markers is limited due to the high cost associated with measuring them and lack of evidence regarding their prognostic value.

The increasing volume of evidence shows that systemic inflammation can be used to predict the prognosis of patients with a variety of cancer types, including breast cancer. ${ }^{7-15}$ Systemic inflammation can be monitored using biochemical or hematological markers, such as elevated C-reactive protein, hypoalbuminemia, and leukocyte, neutrophil, and platelet cell numbers. Many studies have suggested that the levels of inflammation prior to first-line treatment represent prognostic factors in cancer. Among them, the elevation of peripheral blood neutrophil-to-lymphocyte ratio (NLR) and platelet-to-lymphocyte ratio appear to be predictive of poor prognosis in lung cancer, breast cancer, pancreatic cancer, prostate cancer, hepatocellular carcinoma, colorectal cancer, gastric cancer, and endometrial cancer. ${ }^{7-9,16-23}$ The leukocyte minus neutrophil count was considered to be equivalent to the lymphocyte count. The derived NLR (dNLR) was defined as neutrophil count/leukocyte count minus neutrophil count; therefore, the high dNLR may be due to the increased neutrophil count or decreased lymphocyte count. Several other studies have used the dNLR (neutrophil/leukocyte minus neutrophil) as a prognostic indicator; their results have shown that elevated dNLR is associated with poor prognosis of patients with lung cancer, renal cell carcinoma, gastric cancer, hepatocellular carcinoma, colorectal cancer, urothelial carcinoma, pancreatic cancer, and lymphoma. ${ }^{24-30}$ In breast cancer, the prognostic role of dNLR is controversial, particularly in patients with HER2+ breast cancer who have received neoadjuvant chemotherapy. Thus, we aimed to investigate the prognostic role of $\mathrm{dNLR}$ in this patient population.

\section{Materials and methods}

\section{Patients and data collection}

This study was approved by the Ethics Committee of The Third People's Hospital of Bengbu. The Ethics Committee did not require written informed consent be obtained from all patients, as this was a retrospective study, and all data were anonymous. A total of 310 patients who were diagnosed with HER2+ breast cancer and received neoadjuvant chemotherapy in our hospital from May 2006 to November 2013 were retrospectively included in the study. Inclusion criteria were as follows: patients (1) had histologically confirmed HER2+ non-distant metastatic breast cancer and (2) received neoadjuvant chemotherapy followed by radical or breastconserving surgery; and (3) data collection was performed prior to treatment for differential blood counts. Patients with the following conditions were excluded: (1) inflammatory or immune diseases that may affect peripheral blood cell counts, such as diabetes, hypertension, metabolic syndrome, coronary artery disease, kidney disease, and hematological diseases; (2) metastatic or inflammatory breast cancer; (3) use of anti-inflammatory or immunosuppressive drugs prior to enrollment; and (4) infectious disease within 1 month of the study start date.

Data for leukocyte count, neutrophil count, and lymphocyte count, as well as age, tumor size, lymph node metastasis, lymphovascular invasion, histological grade, Breast ImagingReporting and Data System (BI-RADS) classification, and antitumor treatment (eg, surgery, chemotherapy, radiotherapy, and targeted therapy), were collected. Patients received neoadjuvant chemotherapy with four cycles of anthracyclines and cyclophosphamide, and four cycles of paclitaxel with or without herceptin. There were 189 (61\%) patients who received postoperative radiotherapy, while 121 (39\%) patients did not receive radiotherapy. The radiation dose was 50-60 Gy/25-30 fractions.

\section{BI-RADS classification}

We collected BI-RADS data based on the fifth edition of the BI-RADS classification. The definitions for each classification (1-6) are as follows: BI-RADS 6, a confirmed malignant tumor; BI-RADS 5, a tumor highly suggestive of malignancy and requires biopsy; BI-RADS 4, a tumor with suspected malignancy and requires biopsy; BI-RADS 3, a possibly benign tumor with reexamination recommended after 6 months; BI-RADS 2, a benign tumor; BI-RADS 1, tumor-negative.

\section{Treatment and follow-up}

All patients received neoadjuvant chemotherapy followed by surgery. Patients were regularly followed until death or discontinuation from the study. We performed the followup every 3 months after surgery. To assess disease status, ultrasound imaging, MRI, positron emission computed tomography, and computed tomography were used. During the follow-up, we evaluated the extent of disease progression, 
death, and discontinuation. The deadline for follow-up was January 30, 2018. Overall survival (OS) was defined as the time from pathological diagnosis to death, and disease-free survival (DFS) was defined as the time from surgery to the first instance of disease recurrence.

\section{Statistical analysis}

Data analysis was performed using SPSS version 21.0 software (IBM Corporation, Armonk, NY, USA). The dNLR was defined as neutrophil count/leukocyte count minus neutrophil count. Using the receiver operating characteristic (ROC) curve analysis, dNLR with the highest specificity and sensitivity was selected as the cutoff value to discriminate between long and short OS. ${ }^{31,32}$ Patients were divided into two groups based on the dNLR cutoff point: the low-dNLR group (dNLR $<$ cutoff point) and the high-dNLR group (dNLR $\geq$ cutoff point). The relationship between dNLR and clinicopathological features was evaluated by Chi-squared and Fisher exact tests. The Kaplan-Meier method was used to evaluate OS and DFS. The differences in survival between patients from the two groups were compared by log-rank test. The Cox regression model was used for multivariate analysis to verify independent prognostic factors in patients with HER2+ breast cancer. Differences with $P<0.05$ were considered statistically significant.

\section{Results}

\section{Patients' characteristics}

A total of 310 patients were included in the study. Among them, 156 patients underwent breast-conserving surgery and 154 patients underwent modified radical mastectomy. Further, 159 cases were classified as BI-RADS 5, 148 cases were classified as BI-RADS 4, and three cases were classified as BI-RADS 3. All patients received preoperative neoadjuvant chemotherapy. According to the American Joint Committee on Cancer staging system, 42, 128, and 140 cases of patients had stage I, II, and III disease, respectively. There were 34, 152 , and 106 patients with histopathological grade 1,2 , and 3 tumors, respectively, while the histological grade in 18 patients was unknown. The median follow-up time was 62.5 months (9.6-138.5 months). At the end of the follow-up, 270 patients had recurrent or metastatic disease, 246 patients had died, and 16 patients were lost to follow-up.

The average preoperative leukocyte count of the total patient population was $5.2 \pm 1.2\left(10^{9} / \mathrm{L}\right)$. The mean neutrophil count was $3.7 \pm 1.5\left(10^{9} / \mathrm{L}\right)$, and the average dNLR was $2.3 \pm 0.8$. ROC curve analysis showed that the optimal cutoff value for dNLR was 2.7 .

\section{Relationship between dNLR and clinicopathological features}

Table 1 shows the association between dNLR and clinicopathological parameters. Tumor size, disease stage, lymphovascular invasion, and histopathological grade were higher in the high-dNLR group (dNLR $\geq 2.7$ ) than in the low-dNLR group (dNLR $<2.7)(P<0.05)$. Moreover, patients with a higher extent of lymph node metastasis had an increased dNLR $(P=0.052)$.

\section{High dNLR and BI-RADS classification predict poor survival}

The median OS was 44.2 and 69.9 months in patients with high dNLR and low dNLR, respectively $(P<0.001$; Figure 1$)$, while the median DFS in patients with high dNLR and low dNLR was 15.3 and 22.1 months, respectively $(P<0.001$; Figure 2). Patients with BI-RADS classification of 5 had poor OS and DFS compared with those who had BI-RADS classification of 3 or 4 (42.3 vs 71.2 months, $P<0.001$ and

Table I Relationship between dNLR and clinicopathological features in 310 patients with HER2+ breast cancer

\begin{tabular}{|c|c|c|c|}
\hline Parameters & dNLR $<2.7$ & dNLR $\geq 2.7$ & $P$-value \\
\hline$\overline{\text { Age }}$ & $43.5 \pm 12.4$ & $45.2 \pm 10.5$ & 0.812 \\
\hline \multicolumn{4}{|l|}{ Tumor size } \\
\hline $\mathrm{TI}$ & 54 & 4 & \multirow[t]{4}{*}{0.005} \\
\hline $\mathrm{T} 2$ & 120 & 48 & \\
\hline $\mathrm{T} 3$ & 50 & 22 & \\
\hline $\mathrm{T} 4$ & 8 & 4 & \\
\hline \multicolumn{4}{|c|}{ Lymph node metastasis } \\
\hline No & 92 & 20 & \multirow[t]{4}{*}{0.052} \\
\hline NI & 46 & 24 & \\
\hline N2 & 38 & 10 & \\
\hline N3 & 56 & 24 & \\
\hline \multicolumn{4}{|l|}{ AJCC stage } \\
\hline 1 & 38 & 4 & \multirow[t]{3}{*}{0.042} \\
\hline II & 92 & 36 & \\
\hline III & 102 & 38 & \\
\hline \multicolumn{4}{|c|}{ Lymphovascular invasion } \\
\hline Yes & 36 & 26 & \multirow[t]{2}{*}{0.001} \\
\hline No & 196 & 52 & \\
\hline \multicolumn{4}{|c|}{ Pathological type } \\
\hline IDC & 191 & 60 & \multirow[t]{2}{*}{0.293} \\
\hline ILC & 41 & 18 & \\
\hline \multicolumn{4}{|c|}{ Histological grade } \\
\hline I & 32 & 2 & \multirow[t]{4}{*}{$<0.001$} \\
\hline II & 110 & 42 & \\
\hline III & 84 & 22 & \\
\hline Unknown & 8 & 10 & \\
\hline
\end{tabular}

Abbreviations: AJCC, American Joint Committee on Cancer; dNLR, derived neutrophil-to-lymphocyte ratio; IDC, invasive ductal carcinoma; ILC, invasive lobular carcinoma. 


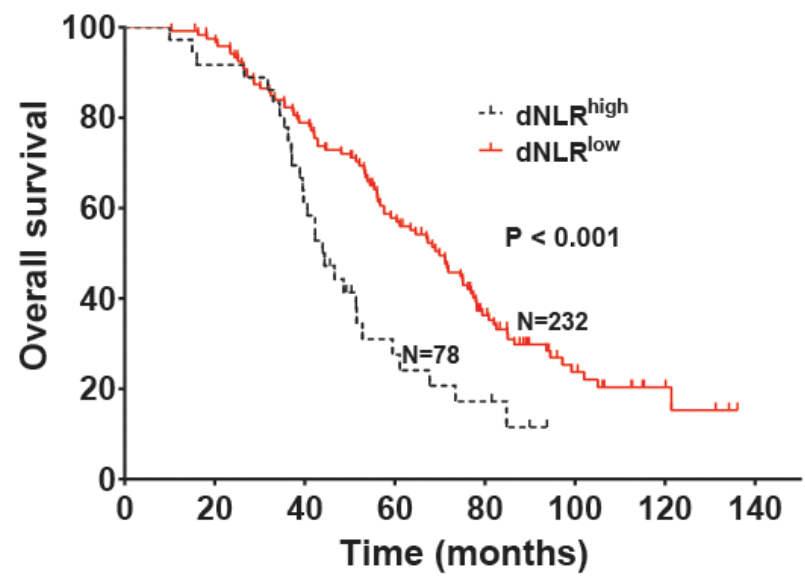

Figure I Overall survival of patients with HER2+ breast cancer stratified by dNLR. Abbreviation: $\mathrm{dNLR}$, derived neutrophil-to-lymphocyte ratio.

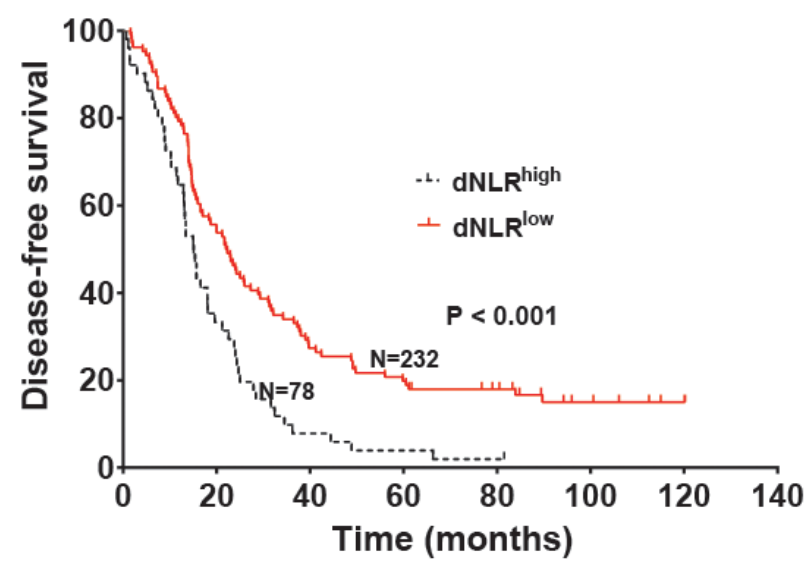

Figure 2 Disease-free survival of patients with HER2+ breast cancer stratified by dNLR.

Abbreviation: $\mathrm{dNLR}$, derived neutrophil-to-lymphocyte ratio.

13.4 vs 23.2 months, $P<0.001$, respectively; Figures 3 and 4). Univariate analysis showed that several patient characteristics, including large tumor size, lymph node metastasis, high-histopathological grade tumors, lymphovascular invasion, high BI-RADS classification, and high dNLR, were correlated with poor OS and DFS $(P<0.001$; Tables 2 and 3). Multivariate analysis showed that elevated dNLR independently predicted poor OS (HR $=1.726,95 \%$ CI: 1.072-2.662, $P=0.009$; Table 2) and DFS (HR $=1.658$, 95\% CI: $1.125-2.426, P=0.026$; Table 3). Furthermore, high BI-RADS classification independently predicted shorter OS (HR $=1.609,95 \%$ CI: 1.216-2.351, $P=0.015$; Table 2$)$ and DFS (HR $=1.925$, 95\% CI: 1.526-2.635, $P=0.021$; Table 3). We found that tumor size and histopathological grade were independent prognostic factors for patients with HER2+ breast cancer.

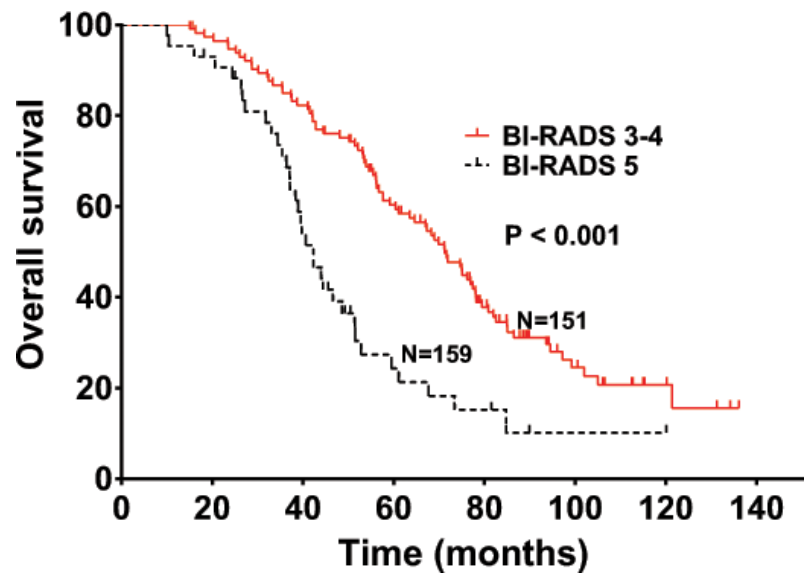

Figure 3 Overall survival of patients with HER2+ breast cancer stratified by $\mathrm{BI}$ RADS classification.

Abbreviation: BI-RADS, Breast Imaging-Reporting and Data System.

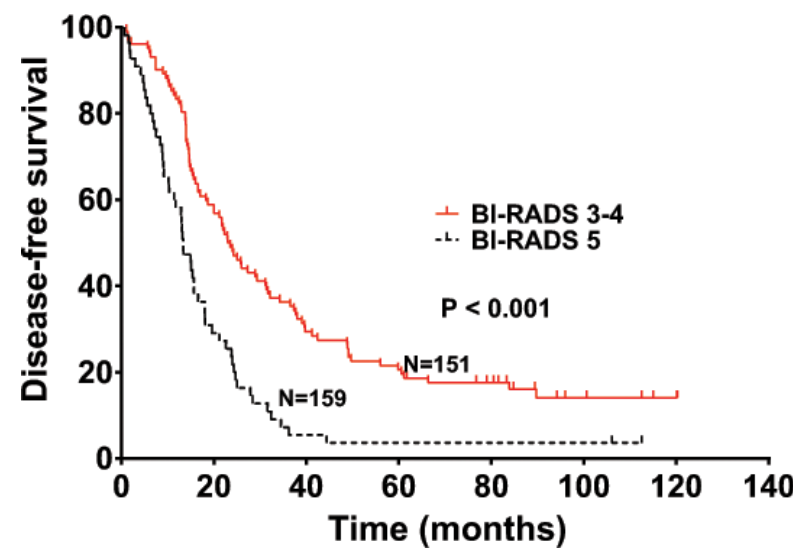

Figure 4 Disease-free survival of patients with HER2+ breast cancer stratified by BI-RADS classification.

Abbreviation: BI-RADS, Breast Imaging-Reporting and Data System.

\section{Discussion}

Several recent studies have shown that systemic inflammation plays an important role in tumorigenesis and disease progression, suggesting the potential utility of inflammation as a prognostic marker. ${ }^{8,916,33-35}$ Tumor-related inflammatory markers include biochemical and hematological indicators such as CA125, and leukocyte, neutrophil, and platelet cell count. Among them, neutrophils promote tumor development by remodeling the extracellular matrix and releasing ROS, nitric oxide, and arginase. ${ }^{36-41}$ Previous reports have shown that neutrophils in circulating blood promote tumor growth and metastasis by producing tumor inflammatory mediators, while lymphocytes inhibit tumor progression through immune surveillance. Notably, tumor lymphocyte infiltration 
Table 2 Univariate and multivariate analyses of OS in HER2+ breast cancer

\begin{tabular}{|c|c|c|c|c|}
\hline \multirow[t]{2}{*}{ Parameters } & \multicolumn{2}{|l|}{ Univariate analysis } & \multicolumn{2}{|c|}{ Multivariate analysis } \\
\hline & HR (95\% Cl) & $P$-value & HR (95\% Cl) & $P$-value \\
\hline \multicolumn{5}{|l|}{ Age (years) } \\
\hline$\geq 50$ vs $<50$ & $1.083(0.73 \mid-1.652)$ & 0.698 & $1.025(0.632-1.686)$ & 0.896 \\
\hline \multicolumn{5}{|l|}{ Tumor size } \\
\hline$\geq 2$ vs $<2$ & $3.542(2.038-5.695)$ & $<0.001$ & $2.623(1.562-4.652)$ & $<0.001$ \\
\hline \multicolumn{5}{|c|}{ Lymph node metastasis } \\
\hline Yes vs no & $2.532(1.815-3.726)$ & $<0.001$ & $1.426(0.897-2.356)$ & 0.132 \\
\hline \multicolumn{5}{|c|}{ Histological grade } \\
\hline $\mathrm{G} 3$ vs $\mathrm{GI} / 2$ & $2.65 I(1.786-3.689)$ & $<0.001$ & $2.013(1.262-3.562)$ & 0.001 \\
\hline \multicolumn{5}{|c|}{ Lymphovascular invasion } \\
\hline Yes vs no & $2.753(1.952-3.856)$ & $<0.001$ & $1.653(0.965-2.124)$ & 0.086 \\
\hline \multicolumn{5}{|l|}{ BI-RADS } \\
\hline 5 vs $3-4$ & $2.285(1.5 \mid 2-3.263)$ & $<0.001$ & $1.609(1.216-2.35 \mathrm{I})$ & 0.015 \\
\hline \multicolumn{5}{|l|}{ dNLR } \\
\hline$\geq 2.5$ vs $<2.5$ & $1.895(1.152-2.862)$ & $<0.001$ & $1.726(1.072-2.662)$ & 0.009 \\
\hline
\end{tabular}

Abbreviations: BI-RADS, Breast Imaging-Reporting and Data System; dNLR, derived neutrophil-to-lymphocyte ratio; OS, overall survival.

Table 3 Univariate and multivariate analyses of DFS in HER2+ breast cancer

\begin{tabular}{|c|c|c|c|c|}
\hline \multirow[t]{2}{*}{ Parameters } & \multicolumn{2}{|l|}{ Univariate analysis } & \multicolumn{2}{|c|}{ Multivariate analysis } \\
\hline & HR (95\% CI) & $P$-value & HR (95\% Cl) & $P$-value \\
\hline \multicolumn{5}{|l|}{ Age (years) } \\
\hline$\geq 50$ vs $<50$ & $0.963(0.925-1.015)$ & 0.562 & $0.826(0.652-1.162)$ & 0.216 \\
\hline \multicolumn{5}{|l|}{ Tumor size } \\
\hline$\geq 2 \mathrm{vs}<2$ & $2.862(1.896-4.562)$ & $<0.001$ & $2.153(1.352-3.653)$ & 0.002 \\
\hline \multicolumn{5}{|c|}{ Lymph node metastasis } \\
\hline Yes vs no & $2.256(1.547-3.256)$ & $<0.001$ & $1.315(0.863-2.153)$ & 0.268 \\
\hline \multicolumn{5}{|c|}{ Histological grade } \\
\hline G3 vs GI/2 & $3.256(2.356-5.125)$ & $<0.001$ & $2.562(1.796-4.152)$ & $<0.001$ \\
\hline \multicolumn{5}{|c|}{ Lymphovascular invasion } \\
\hline Yes vs no & $2.956(2.036-4.352)$ & $<0.001$ & $2.263(1.265-2.965)$ & 0.001 \\
\hline \multicolumn{5}{|l|}{ BI-RADS } \\
\hline 5 vs $3-4$ & $2.562(1.756-3.562)$ & $<0.001$ & $1.925(1.526-2.635)$ & 0.021 \\
\hline \multicolumn{5}{|l|}{$\mathrm{dNLR}$} \\
\hline$\geq 2.5 \mathrm{vs}<2.5$ & $1.856(1.325-2.976)$ & $<0.001$ & $1.658(1.125-2.426)$ & 0.026 \\
\hline
\end{tabular}

Abbreviations: BI-RADS, Breast Imaging-Reporting and Data System; DFS, disease-free survival; dNLR, derived neutrophil-to-lymphocyte ratio.

appears to be related to tumor prognosis..$^{5,6,42}$ T-cell activation status was positively associated with breast cancer patients' OS. ${ }^{32,43}$ Besides, PD-L1+ tumor-infiltrating lymphocytes status showed an independent favorable prognostic value in patients with inflammatory breast cancer, suggesting that immune checkpoint immunotherapy should be explored and correlated with prognosis in these patients. ${ }^{44}$

Several studies have shown that high dNLR was associated with poor prognosis in multiple cancer types. ${ }^{28,45-48}$ Among these, a few have investigated the role of dNLR in breast cancer; however, the results were inconsistent. Proctor et $\mathrm{al}^{49}$ found that elevated dNLR was associated with poor prognosis in patients with breast cancer; however, this study did not take into account the clinical stage, tumor histopathological grade, hormone receptor status, and previous treatments received by the patients, thus making it impossible to assess whether dNLR was associated with prognosis post adjustment of other factors. In another study, Dirican et $\mathrm{al}^{47}$ analyzed 1,527 cases of breast cancer and found a significant association between elevated dNLR and DFS and OS; however, their multivariate analysis did not show that dNLR could be used as an independent prognostic indicator for breast cancer. Although the sample size of this study was relatively large, it had a short follow-up period, necessitating further verification.

Our study provides evidence that dNLR is significantly associated with OS and DFS in patients with HER2+ breast cancer. This correlation with OS and DFS remained 
significant after adjusting for patients' age, tumor size, lymph node metastasis, and histopathological grading. Our results suggest that elevated dNLR is independently correlated with high mortality, suggesting its potential utility as an independent prognostic indicator for patients with HER2+ breast cancer. In our study, patients with breast cancer underwent routine blood tests prior to first-line treatment. Consequently, the assessment of dNLR was readily available without any additional costs. Therefore, preoperative dNLR may be used as an indicator to predict the survival of patients with HER2+ breast cancer. Moreover, we found that increased BI-RADS classification independently correlated with OS and DFS.

Few studies have reported on the relationship between dNLR and the prognosis of patients with HER2+ breast cancer, particularly in the Chinese population. To our knowledge, our study had the largest sample size compared with any other studies that investigated the prognostic value of dNLR in Chinese patients with HER2+ breast cancer. However, our study also had some limitations. First, selection bias in the retrospective single-center study was inevitable. Second, there was no external validation used in our study. Despite these caveats, our study still provides strong evidence for the prognostic role of $\mathrm{dNLR}$ and BI-RADS classification in patients with HER2+ breast cancer who have received neoadjuvant chemotherapy.

\section{Conclusion}

This study shows that dNLR and BI-RADS classification may represent independent prognostic factors in patients with HER2 + breast cancer who have received neoadjuvant chemotherapy. Prospective studies with a large sample are still needed to confirm the prognostic value of these factors.

\section{Disclosure}

The authors report no conflicts of interest in this work.

\section{References}

1. Fan L, Strasser-Weippl K, Li JJ, et al. Breast cancer in China. Lancet Oncol. 2014;15(7):e279-e289.

2. Zhu X, Ying J, Wang F, Wang J, Yang H. Estrogen receptor, progesterone receptor, and human epidermal growth factor receptor 2 status in invasive breast cancer: a 3,198 cases study at National Cancer Center, China. Breast Cancer Res Treat. 2014;147(3):551-555.

3. Sparano JA, Gray RJ, Makower DF, et al. Prospective Validation of a 21-Gene Expression Assay in Breast Cancer. $N$ Engl J Med. 2015;373(21):2005-2014.

4. Ali HR, Provenzano E, Dawson SJ, et al. Association between CD8+ T-cell infiltration and breast cancer survival in 12,439 patients. Ann Oncol. 2014;25(8):1536-1543.
5. Muenst S, Soysal SD, Gao F, Obermann EC, Oertli D, Gillanders WE. The presence of programmed death 1 (PD-1)-positive tumor-infiltrating lymphocytes is associated with poor prognosis in human breast cancer. Breast Cancer Res Treat. 2013;139(3):667-676.

6. Mahmoud SM, Paish EC, Powe DG, et al. An evaluation of the clinical significance of FOXP3 + infiltrating cells in human breast cancer. Breast Cancer Res Treat. 2011;127(1):99-108.

7. Liu C, Huang Z, Wang Q, et al. Usefulness of neutrophil-to-lymphocyte ratio and platelet-to-lymphocyte ratio in hormone-receptor-negative breast cancer. Onco Targets Ther. 2016;9:4653-4660.

8. Koh CH, Bhoo-Pathy N, Ng KL, et al. Utility of pre-treatment neutrophil-lymphocyte ratio and platelet-lymphocyte ratio as prognostic factors in breast cancer. Br J Cancer. 2015;113(1):150-158.

9. Yao M, Liu Y, Jin H, et al. Prognostic value of preoperative inflammatory markers in Chinese patients with breast cancer. Onco Targets Ther. 2014;7:1743-1752.

10. Noh H, Eomm M, Han A. Usefulness of pretreatment neutrophil to lymphocyte ratio in predicting disease-specific survival in breast cancer patients. J Breast Cancer. 2013;16(1):55-59.

11. Yamagishi T, Fujimoto N, Nishi H, et al. Prognostic significance of the lymphocyte-to-monocyte ratio in patients with malignant pleural mesothelioma. Lung Cancer. 2015;90(1):111-117.

12. Spolverato G, Maqsood H, Kim Y, et al. Neutrophil-lymphocyte and platelet-lymphocyte ratio in patients after resection for hepato-pancreatico-biliary malignancies. J Surg Oncol. 2015;111(7):868-874.

13. Peng B, Wang YH, Liu YM, Ma LX. Prognostic significance of the neutrophil to lymphocyte ratio in patients with non-small cell lung cancer: a systemic review and meta-analysis. Int J Clin Exp Med. 2015;8(3):3098-3106.

14. Ferrucci PF, Gandini S, Battaglia A, et al. Baseline neutrophilto-lymphocyte ratio is associated with outcome of ipilimumabtreated metastatic melanoma patients. Br J Cancer. 2015;112(12): 1904-1910.

15. Pinato DJ, Shiner RJ, Seckl MJ, Stebbing J, Sharma R, Mauri FA. Prognostic performance of inflammation-based prognostic indices in primary operable non-small cell lung cancer. $B r J$ Cancer. 2014;110(8):1930-1935.

16. Pistelli M, De Lisa M, Ballatore Z, et al. Pre-treatment neutrophil to lymphocyte ratio may be a useful tool in predicting survival in early triple negative breast cancer patients. BMC Cancer. 2015;15:195.

17. Stotz M, Gerger A, Eisner F, et al. Increased neutrophil-lymphocyte ratio is a poor prognostic factor in patients with primary operable and inoperable pancreatic cancer. Br J Cancer. 2013;109(2):416-421.

18. Lorente D, Mateo J, Templeton AJ, et al. Baseline neutrophil-lymphocyte ratio (NLR) is associated with survival and response to treatment with second-line chemotherapy for advanced prostate cancer independent of baseline steroid use. Ann Oncol. 2015;26(4):750-755.

19. Kinoshita A, Onoda H, Imai N, et al. Comparison of the prognostic value of inflammation-based prognostic scores in patients with hepatocellular carcinoma. Br J Cancer. 2012;107(6):988-993.

20. Li MX, Liu XM, Zhang XF, et al. Prognostic role of neutrophil-tolymphocyte ratio in colorectal cancer: a systematic review and metaanalysis. Int J Cancer. 2014;134(10):2403-2413.

21. Liu C, Wu S, Meng X, et al. Predictive value of peripheral regulatory $\mathrm{T}$ cells in non-small cell lung cancer patients undergoing radiotherapy. Oncotarget. 2017;8(26):43427-43438.

22. Xin-Ji Z, Yong-Gang L, Xiao-Jun S, Xiao-Wu C, Dong Z, Da-Jian Z. The prognostic role of neutrophils to lymphocytes ratio and platelet count in gastric cancer: A meta-analysis. Int J Surg. 2015;21:84-91.

23. Cummings M, Merone L, Keeble C, et al. Preoperative neutrophil: lymphocyte and platelet:lymphocyte ratios predict endometrial cancer survival. Br J Cancer. 2015;113(2):311-320.

24. Song S, Li C, Li S, Gao H, Lan X, Xue Y. Derived neutrophil to lymphocyte ratio and monocyte to lymphocyte ratio may be better biomarkers for predicting overall survival of patients with advanced gastric cancer. Onco Targets Ther. 2017;10:3145-3154. 
25. Ferrucci PF, Ascierto PA, Pigozzo J, et al. Baseline neutrophils and derived neutrophil-to-lymphocyte ratio: prognostic relevance in metastatic melanoma patients receiving ipilimumab. Ann Oncol. 2016;27(4):732-738.

26. Grenader T, Nash S, Plotkin Y, et al. Derived neutrophil lymphocyte ratio may predict benefit from cisplatin in the advanced biliary cancer: the ABC-02 and BT-22 studies. Ann Oncol. 2015;26(9):1910-1916.

27. Deng Q, He B, Liu X, et al. Prognostic value of pre-operative inflammatory response biomarkers in gastric cancer patients and the construction of a predictive model. J Transl Med. 2015;13:66.

28. Absenger G, Szkandera J, Pichler M, et al. A derived neutrophil to lymphocyte ratio predicts clinical outcome in stage II and III colon cancer patients. Br J Cancer. 2013;109(2):395-400.

29. Dalpiaz O, Luef T, Seles M, et al. Critical evaluation of the potential prognostic value of the pretreatment-derived neutrophil-lymphocyte ratio under consideration of $\mathrm{C}$-reactive protein levels in clear cell renal cell carcinoma. Br J Cancer. 2017;116(1):85-90.

30. Russo A, Franchina T, Ricciardi GRR, et al. Baseline neutrophilia, derived neutrophil-to-lymphocyte ratio (dNLR), platelet-to-lymphocyte ratio (PLR), and outcome in non small cell lung cancer (NSCLC) treated with Nivolumab or Docetaxel. J Cell Physiol. 2018;233(10): 6337-6343.

31. Yang JC, Risch E, Zhang M, Huang C, Huang H, Lu L. Association of tRNA methyltransferase NSUN2/IGF-II molecular signature with ovarian cancer survival. Future Oncol. 2017;13(22):1981-1990.

32. Lu L, Bai Y, Wang Z. Elevated T cell activation score is associated with improved survival of breast cancer. Breast Cancer Res Treat. 2017;164(3):689-696.

33. Liu C, Wang Q, Sun B, et al. Low BMI is correlated with increased TGF- $\beta$ and IL-10 mRNA levels in the peripheral blood of breast cancer patients. IUBMB Life. 2018;70(3):237-245.

34. Jia W, Wu J, Jia H, et al. The Peripheral Blood Neutrophil-To-Lymphocyte Ratio Is Superior to the Lymphocyte-To-Monocyte Ratio for Predicting the Long-Term Survival of Triple-Negative Breast Cancer Patients. PLoS One. 2015;10(11):e0143061.

35. Liu C, Wang W, Meng X, et al. Albumin/globulin ratio is negatively correlated with PD-1 and CD25 mRNA levels in breast cancer patients. Onco Targets Ther. 2018;11:2131-2139.

36. Allen MD, Jones LJ. The role of inflammation in progression of breast cancer: Friend or foe? (Review). Int J Oncol. 2015;47(3):797-805.
37. Hattar K, Franz K, Ludwig M, et al. Interactions between neutrophils and non-small cell lung cancer cells: enhancement of tumor proliferation and inflammatory mediator synthesis. Cancer Immunol Immunother. 2014;63(12):1297-1306.

38. Diakos CI, Charles KA, McMillan DC, Clarke SJ. Cancerrelated inflammation and treatment effectiveness. Lancet Oncol. 2014;15(11):e493-e503.

39. Gay LJ, Felding-Habermann B. Contribution of platelets to tumour metastasis. Nat Rev Cancer. 2011;11(2):123-134.

40. Mantovani A, Allavena P, Sica A, Balkwill F. Cancer-related inflammation. Nature. 2008;454(7203):436-444.

41. De Larco JE, Wuertz BR, Furcht LT. The potential role of neutrophils in promoting the metastatic phenotype of tumors releasing interleukin-8. Clin Cancer Res. 2004;10(15):4895-4900.

42. Tao H, Mimura Y, Aoe K, et al. Prognostic potential of FOXP3 expression in non-small cell lung cancer cells combined with tumor-infiltrating regulatory T cells. Lung Cancer. 2012;75(1):95-101.

43. Shao S, Risch E, Burner D, Lu L, Minev B, Ma W. IFN $\gamma$ enhances cytotoxic efficiency of the cytotoxic $\mathrm{T}$ lymphocytes against human glioma cells. Int Immunopharmacol. 2017;47:159-165.

44. Arias-Pulido H, Cimino-Mathews A, Chaher N, et al. The combined presence of $\mathrm{CD} 20+\mathrm{B}$ cells and PD-L1+tumor-infiltrating lymphocytes in inflammatory breast cancer is prognostic of improved patient outcome. Breast Cancer Res Treat. 2018;171(2):273-282.

45. Cox S, Hurt C, Grenader T, Mukherjee S, Bridgewater J, Crosby T. The prognostic value of derived neutrophil to lymphocyte ratio in oesophageal cancer treated with definitive chemoradiotherapy. Radiother Oncol. 2017;125(1):154-159.

46. Krenn-Pilko S, Langsenlehner U, Stojakovic T, et al. The elevated preoperative derived neutrophil-to-lymphocyte ratio predicts poor clinical outcome in breast cancer patients. Tumour Biol. 2016;37(1):361-368

47. Dirican A, Kucukzeybek BB, Alacacioglu A, et al. Do the derived neutrophil to lymphocyte ratio and the neutrophil to lymphocyte ratio predict prognosis in breast cancer? Int J Clin Oncol. 2015;20(1):70-81.

48. Ying HQ, Deng QW, He BS, et al. The prognostic value of preoperative NLR, d-NLR, PLR and LMR for predicting clinical outcome in surgical colorectal cancer patients. Med Oncol. 2014;31(12):305.

49. Proctor MJ, McMillan DC, Morrison DS, Fletcher CD, Horgan PG, Clarke SJ. A derived neutrophil to lymphocyte ratio predicts survival in patients with cancer. Br J Cancer. 2012;107(4):695-699.
Cancer Management and Research

\section{Publish your work in this journal}

Cancer Management and Research is an international, peer-reviewed open access journal focusing on cancer research and the optimal use of preventative and integrated treatment interventions to achieve improved outcomes, enhanced survival and quality of life for the cancer patient. The manuscript management system is completely online and includes

\section{Dovepress}

a very quick and fair peer-review system, which is all easy to use. Visit http://www.dovepress.com/testimonials.php to read real quotes from published authors. 